Chinese Journal of Astronomy and Astrophysics manuscript no.

(LTEX: ms.tex; printed on September 14, 2021; 17:34)

\title{
Simultaneous multi-wavelength observations of the TeV Blazar Mrk 421 during February - March 2003: X-ray and NIR correlated variability
}

\author{
Alok C. Gupta ${ }^{1 \star}$, B. S. Acharya ${ }^{2}$, Debanjan Bose ${ }^{2}$, Varsha R. \\ Chitnis ${ }^{2}$ and Jun-Hui Fan ${ }^{1}$ \\ 1 Center for Astrophysics, Guangzhou University, Guangzhou 510006, China \\ 2 Tata Institute of Fundamental Research, Homi Bhabha Road, Colaba, \\ Mumbai - 400 005, India
}

\begin{abstract}
In the present paper, we have reported the result of simultaneous multi-wavelength observations of the TeV blazar Mrk 421 during February - March 2003. In this period, we have observed Mrk 421 using Pachmarhi Array of Cerenkov Telescopes (PACT) of Tata Institute of Fundamental Research at Pachmarhi, India. Other simultaneous data were taken from the published literature and public data archives. We have analyzed the high quality X-ray $(2-20 \mathrm{keV})$ observations from the NASA Rossi X-Ray Timing Explorer (RXTE). We have seen a possible correlated variability between X-ray and J band $(1.25 \mu)$ near infrared (NIR) wavelength. This is the first case of X-ray and NIR correlated variability in Mrk 421 or any high energy peaked (HBL) blazar. The correlated variability reported here is indicating a similar origin for NIR and X-ray emission. The emission is not affected much by the environment of the surrounding medium around the central engine of the Mrk 421. The observations are consistent with the shock-in-jet model for the emission of radiations.
\end{abstract}

Key words: galaxies: active - galaxies: blazars: general - galaxies: blazars: individual: Mrk 421

\section{INTRODUCTION}

A small subgroup of radio-loud active galactic nuclei (AGNs) show significant flux variability in the complete electromagnetic (EM) spectrum, variable polarization and their radiation at all wavelengths is predominantly non-thermal. They are known as blazars, which is a collective name of subclasses (BL Lac objects, optically violent variables OVVs, high polarization quasars HPQs and flat spectrum radio quasars FSRQs) of radio-loud AGNs. On a unified model of radioloud AGNs based on the angle between the line of sight and the emitted jet from the source, blazars jet make angle of $<10^{\circ}$ from the line of sight (Urry

$\star$ E-mail: acgupta30@gmail.com 
\& Padovani 1995). Since, blazars emit radiation in the complete EM spectrum which gives an excellent opportunity to study the spectral energy distribution (SEDs). It is found from observations that blazars SEDs have two peaks. The first component peaks any where from IR to optical in so called red blazars or low energy blazars (LBLs) or radio selected blazars (RBLs) and at UV/X-ray in so called blue blazars or high energy blazars (HBLs) or X-ray selected blazars (XBLs). Its origin is synchrotron emission from high energy electrons in the jet. The second component extends up to $\gamma$-rays, peaking at GeV energies in RBLs and at $\mathrm{TeV}$ in XBLs. The electromagnetic emission is dominated by synchrotron component at low-energy and at high-energy by inverse Compton component (Coppi 1999, Sikora et al. 2001, Krawczynski 2004).

Mrk 421 is the nearest detected TeV BL Lac object (redshift $\mathrm{z}=0.031$ ). It was first noted to be an object with blue excess which later turned out to be an elliptical galaxy with bright point like nucleus (Ulrich et al. 1975). Since the energy of synchrotron peak of the source is higher than $0.1 \mathrm{keV}$, it is classified as a HBL. Mrk 421 was the first extragalactic object discovered at TeV energies (Punch et al. 1992). This source was later confirmed by the high energy gamma ray astronomy (HEGRA) group (Petry et al. 1996). It is also one of the $\mathrm{TeV}$ blazars detected by energetic gamma ray experiment telescope (EGRET) instrument in the $30 \mathrm{MeV}-30 \mathrm{GeV}$ energy range by the Compton gamma ray observatory (CGRO) (Thompson et al. 1995). This source has been detected by the other detectors like, the imaging Compton telescope (COMPTEL) on board CGRO at the $3.2 \sigma$ level in the $10-30 \mathrm{MeV}$ energy range (Collmar et al. 1999) and the solar tower atmosphere Čerenkov effect experiment (STACEE) in the 140 $\mathrm{GeV}$ energy band (Boone et al. 2002).

Mrk 421 variability has been studied in all EM regimes in isolation. An exhaustive compilation of radio data at 22 and $37 \mathrm{GHz}$, spanning for about 25 years, for several extragalactic sources including Mrk 421 were reported by (Taräsranta et al. 2004, 2005). NIR data for three decades, for several blazars including Mrk 421, were given by (Fan \& Lin 1999). A much systematic and comprehensive study of this source was done by Gupta et al. (2004) in the same period of the campaign for which the present paper is written. In the compiled optical data for long term observations, variation of $4.6 \mathrm{mag}$ was reported by Stein et al. (1976) and rapid variability of $1.4 \mathrm{mag}$ in 2.5 hours was reported by Xie et al. (1988). There are several simultaneous X-ray and gamma-ray as well as multiwavelength campaigns for the source (Makino et al. 1987, Macomb et al. 1995, Takahashi et al. 2000, Katarzynski et al. 2003, Blazejowski et al. 2005).

In the present paper, we aimed to search for correlated multi-wavelength variability in Mrk 421. This kind of study will be an important tool for understanding the emission mechanism of blazars. This paper is structured as follows. In section 2, we present multi-wavelength observations and data reduction; section 3 and 4 give the results and discussions of the present work.

\section{OBSERVATIONS, DATA AND THE DATA REDUCTION}

\subsection{TeV Observations with PACT}

We have used Pachmarhi Array of Čerenkov Telescopes (PACT), for observation of Mrk 421 in TeV gamma rays. PACT is located in Central India (latitude $22^{\circ}$ $28^{\prime} \mathrm{N}$, longitude $78^{\circ} 25^{\prime} \mathrm{E}$, altitude $1075 \mathrm{~m}$ ). We use wavefront sampling technique to detect $\mathrm{TeV} \gamma$-rays from astronomical sources. There are 24 telescopes spread over an area of $80 \mathrm{~m} \times 100 \mathrm{~m}$. Each telescope has 7 para-axially mounted parabolic mirrors (f/d $\sim 1$ ) of diameter $0.9 \mathrm{~m}$ with a PMT (EMI 9807B) mounted at the focus of each mirror. Each telescope is independently steerable, controlled 


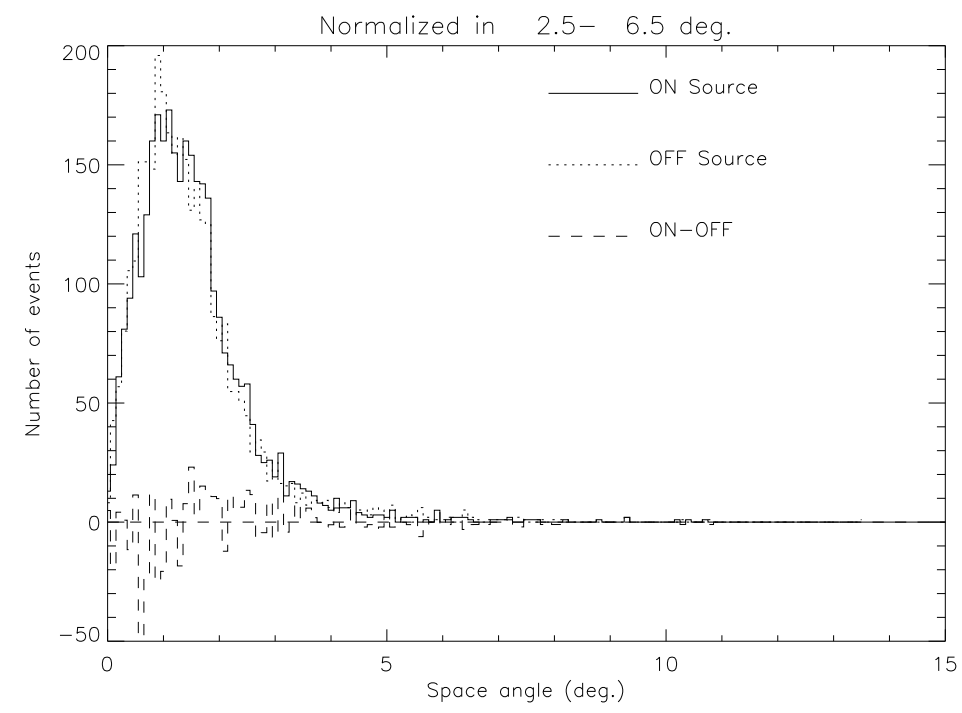

Fig. 1 Space-angle distribution of source and background events for a typical run.

remotely and monitored throughout the observation (Gothe et al. 2000). The entire array is sub-divided into 4 sectors with 6 telescopes in each. Each sector has its own data acquisition system (DAQ) where data on real time, relative arrival time of PMT pulses (using TDCs) and photon density (using ADCs) are recorded. A Master DAQ at the center of the array is also used for recording informations of an event relevant to entire array. The 7 PMT pulses of a telescope are also linearly added to form a telescope pulse for trigger generation. Data recording is initiated when a coincidence of 4 out of any 6 telescope pulses generates an event trigger for a sector. Typical trigger rate was about 1-3 Hz per sector. The details of this array are given in (Bhat et al. 2000, Majumdar et al. 2003).

Observations are carried out in ON-OFF mode on clear moonless nights. In year 2003 from 26th February to 5th March there was a world-wide multiwavelength campaign in several wavebands for this source including PACT. During these nights 2 sectors out of 4 were aligned along the source direction and remaining 2 were looking at a background region simultaneously. The background region is chosen to be a dark region with the same declination as that of the source but with different RA. Background region is chosen in such a way that there is substantial overlap of zenith angle range between the source and background runs. The typical run span was about 1-3 hours. The sectors that look at source and background were interchanged on a daily basis.

A number of preliminary checks were carried out on the data before doing actual analysis. It was found that data taken on 26th and 27th February, 3rd and 5th March were very bad therefore rejected. Observations taken on 28th February, 1st, 2nd and 4th March are analyzed. The arrival direction of each shower is determined by reconstructing shower front using the relative arrival times of Čerenkov photons at various telescopes (or PMTs). Čerenkov photon front is then fitted with a plane, normal to this plane gives the direction of the shower axis. Then, for each shower or event, this space angle is estimated as an angle between the direction of shower axis and the source direction. Thus space angles 
are obtained for all the events for source as well as background runs. Space angle distributions of source runs are compared with respective background runs over the same zenith angle region. Due to some technical problem same night's source and background runs could not be compared for these runs. Each source run is compared with previous nights or next nights background run so that the geometry of the telescope setup is also same for Source and Background runs. For this comparison, the shapes of space angle distributions in $2.5^{\circ}$ to $6.5^{\circ}$ region of source and background were normalized, as we do not expect any signal beyond $2.5^{\circ}$ (Majumdar et al. 2003). Normalization of distributions corresponding to the background with that of the source is necessary as these two data sets were taken at different times. Differences between the number of source and normalized background events within $2.5^{\circ}$ gives the estimate of $\gamma$-ray events. Figure 1 shows the space angle distributions for a typical pair of source and background runs. Details of analysis procedure are given in (Bose et al. 2005). During the campaign nights no excess of events over the background is detected in any of those four nights, implying $\gamma$-ray flux is close to or below the sensitivity limit of PACT.

Čerenkov photon showers initiated by $\gamma$-rays and protons were simulated using CORSIKA air shower simulation (Heck et al. 1998) code to estimate trigger rate, energy threshold, collection area etc for the PACT setup. For $\gamma$-rays incident vertically the energy threshold, defined as the peak of differential rate curve, is estimated to be $750 \mathrm{GeV}$ and the corresponding collection area is $1.58 \times 10^{5} \mathrm{~m}^{2}$. For Mkn421, which is at an angle of $20^{\circ}$ w.r.t. zenith the energy threshold is estimated to be $1.2 \mathrm{TeV}$ and the collection area as $1.8 \times 10^{5} \mathrm{~m}^{2}$.

\subsection{X-Ray Observations with RXTE}

We have analyzed Mrk 421 data observed with RXTE during 26/2/2003 $6 / 3 / 2003$. We have extracted archival data sets corresponding to this multiwavelength campaign under the guest observing program 80172. RXTE has two types of detectors viz, Proportional Counter Array (PCA) and High-Energy Xray Timing Explorer (HEXTE) on-board along with All Sky Monitor (ASM). The PCA consists of five identical xenon filled proportional counter units (PCUs) covering an energy range of 2-60 keV. During these observations only PCU 0 and PCU 2 were used. Since PCU 0 lost pressure in the top veto at the beginning of Epoch 5, we have used only data from PCU 2. HEXTE consists of two clusters of phoswich scintillation detectors covering an energy range of $15-250 \mathrm{keV}$, but is less sensitive. We do not discuss HEXTE data here. ASM consists of three xenon filled position sensitive proportional counters with field of view of $6 \times 90$ degrees. It covers $80 \%$ of the sky every 90 minutes and spans an energy range of 2-10 keV.

We analyzed Standard 2 PCA data which has a time resolution of 16s with energy information in 128 channels. Data reduction is done with FTOOLS (version 5.3.1) distributed as part of HEASOFT (version 5.3). For each of the observations, data was filtered using standard procedure for faint sources given in RXTE Cook Book. For extraction of background, model appropriate for faint sources (pca_bkgd_cmfaintl7_eMv20031123.mdl) was used. Light curves were extracted from data for three energy bands: 2-9, 9-20 and 20-40 keV. Background light

curves were also extracted and subtracted from source light curves. We obtained ASM data from MIT archive. Light curves were generated taking one-day average. 


\subsection{Data from Literature: Multi-wavelength Data}

Near Infrared data in $\mathrm{J}$ band used in the present paper is taken from (Gupta et al. 2004). They have done observations from 1.2 meter optical/NIR telescope at Gurushikhar observatory, Mount Abu, India using NICMOS-3 detector and $\mathrm{J}$ band filter. The detail about NIR observations and data reductions is given in (Gupta et al. 2004).

The radio data is taken from the recent paper by (Taräsranta et al. 2005). They observed the source during the campaign by their 17.7 meter Metsähovi radio telescope at 22 and $37 \mathrm{GHz}$. The detail about radio data is given in (Taräsranta et al. 1998).

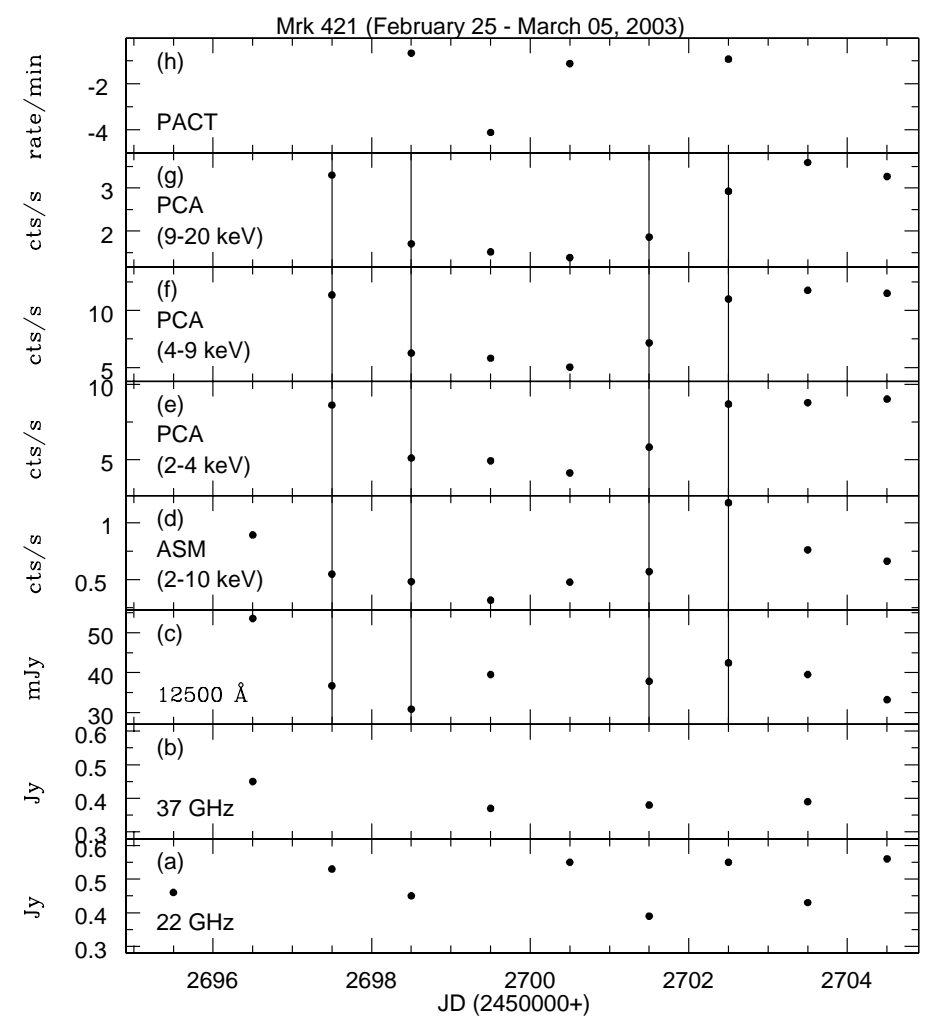

Fig. 2 Multi-wavelength data of Mrk 421 as a function of time for all bands from radio to gamma-rays observed during February 25 - March 05,2003 . Vertical lines in panel (c) $-(\mathrm{g})$ show simultaneous variability in NIR and X-ray bands. In general uncertainties are smaller than the symbols, the error bars have been omitted.

\section{RESULTS}

\subsection{Multi-wavelength Light Curves}

Figure 2 gives the radio to gamma-ray light curves for the multi-wavelength campaign during February 25 - March 05, 2003. The data plotted here for different bands of the EM spectrum is daily average. Daily average of a specific date is 


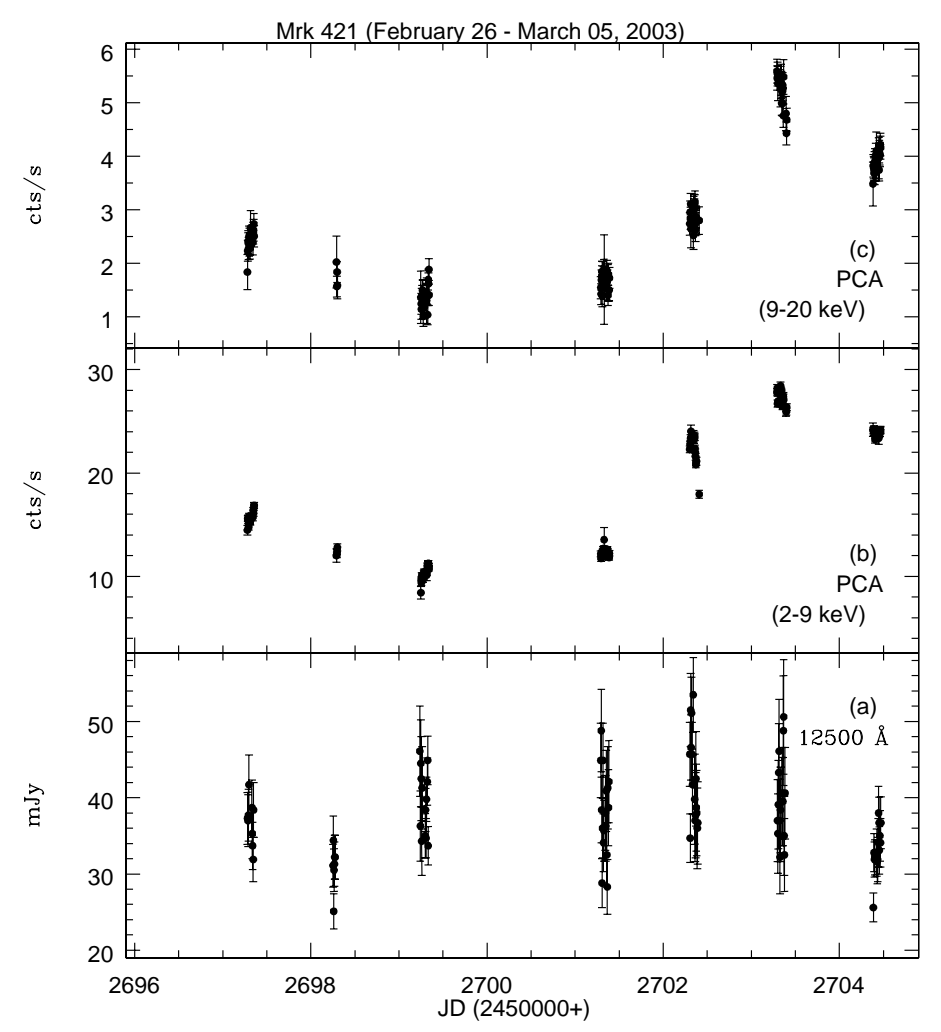

Fig. 3 NIR and X-ray bands data of Mrk 421 as a function of time observed during February 26 - March 05, 2003.

reported at $00 \mathrm{~h} 00 \mathrm{~m} 00 \mathrm{~s}$ UT. The radio flux seems to be in stable state, implies variability timescale may be longer than the duration of the campaign. On the other hand gamma-ray data is noisy. The figure shows highly correlated variability among the different energy bands of the PCA data.

Figure 3 gives the NIR and X-ray light curves (with 5 minutes binning) for the observations during February 26 - March 05, 2003. X-ray coverage was much longer than the NIR coverage therefore we have selected that portion of X-ray data which was approximately simultaneous with NIR. For this plot PCA data of 2 energy bands, $2-4 \mathrm{keV}$ and $4-9 \mathrm{keV}$ are combined.

\subsection{Cross correlation function (ZDCF)}

We computed ZDCF (Z-transformed discrete correlation function) (Alexander 1997) from light curves in X-ray and NIR bands. The ZDCF is a method for determining the cross-correlation function (CCF) of light curves in different energy bands which have non-evenly sampled data. The ZDCF makes use of the Fisher's z-transform of the correlation coefficient. Fisher's z-transform of the linear correlation coefficient, $r$, is used to estimate the confidence level of the measured correlation. This method attempts to correct the biases that affect the original DCF (discrete correlation function) by using equal population binning. The ZDCF involves the following three steps:

(i) All possible pairs of observations, $\left(\mathrm{a}_{i}, \mathrm{~b}_{j}\right)$, are sorted according to their time- 

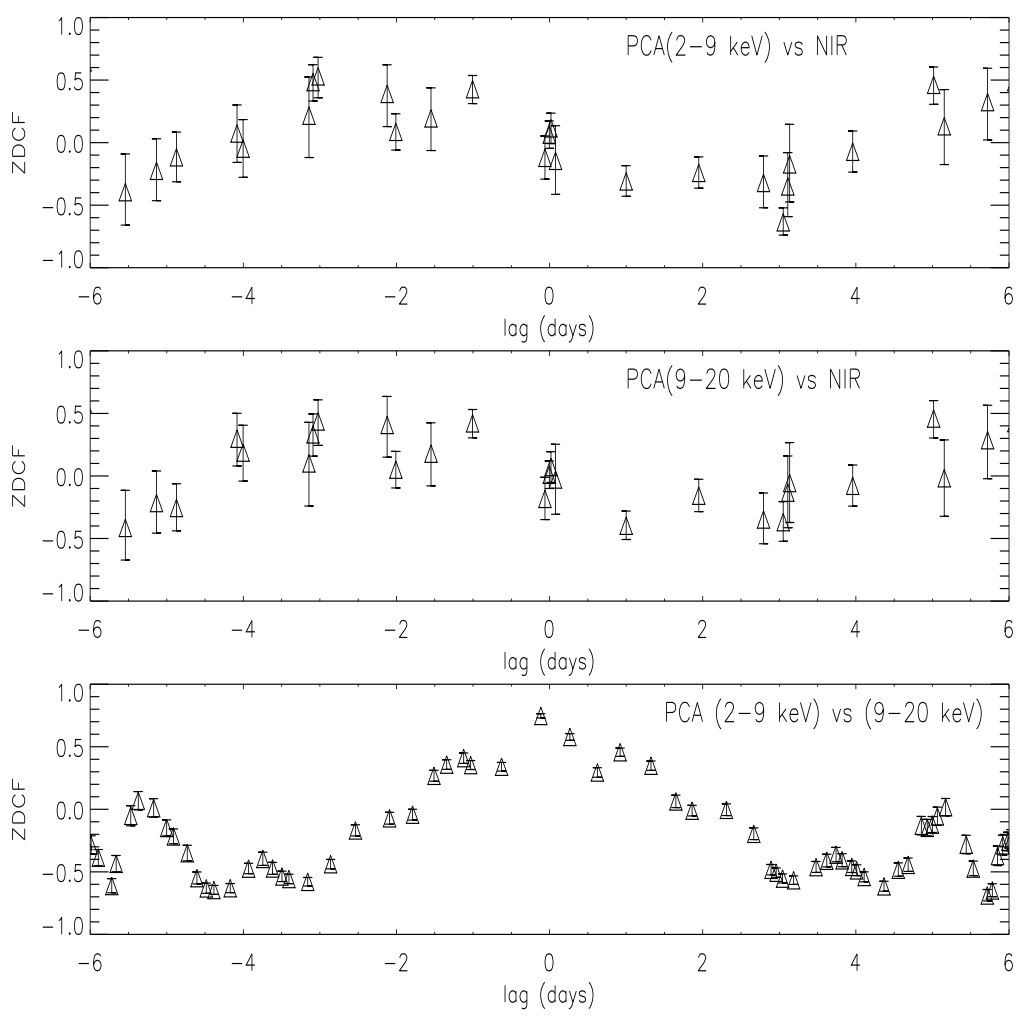

Fig. 4 NIR and X-ray correlations. Positive lags imply that the second light curve lags the first.

lag $t_{i}-t_{j}$, and binned into equal population bins.

(ii) Each bin is assigned its mean time-lag and the intervals above and below the mean that contain $1 \sigma$ of the each point.

(iii) The correlation coefficients of the bins are calculated and z-transformed. The error is calculated in $\mathrm{z}$-space and transformed back to r-space.

The time-lag corresponding to the ZDCF is assumed as the time delay between both components. This function is much more efficient in detecting any correlation present also it takes care of the data gaps. The ZDCF seems to peak at a negative lag -3 days, which implies that X-ray variability lags the NIR variability. Since the dataset is sparse and the value of ZDCF (max) is $\sim 0.5$, so, we claim it as a weak correlated variability. It will be interesting to see with more such observations with focused effort in future.

The ZDCFs are plotted in figure 4 for the three combination of light curves plotted in figure 3. The cross-correlation coefficient $\max (\mathrm{ZDCF})$ and time lag $\tau$ for each combination of light curves are as follows:

(i) For PCA $(2-9 \mathrm{keV})$ vs $12500 \stackrel{\AA}{A}$

$$
\max (\mathrm{ZDCF})=0.530_{-0.1515}^{+0.1715}, \tau=-3.02 \text { days }
$$

(ii) For PCA $(9-20 \mathrm{keV})$ vs $12500 \AA$

$\max (\mathrm{ZDCF})=0.460_{-0.1728}^{+0.1915}, \tau=-3.02$ days

(iii) For PCA $(2-9 \mathrm{keV})$ vs PCA $(9-20 \mathrm{keV})$

$\max (\mathrm{ZDCF})=0.747_{-0.0168}^{+0.0173}, \tau=-0.11$ days 
The visual inspection of the data in the figure 2 show, a strong correlation in NIR and X-ray bands at JD 2452697.5, 2452698.5, 2452701.5 and 2452702.5. In particular, the source has tendency to come to faint stage at JD 2452697.5 and a flaring activity at JD 2452701.5. Thus the positive correlation is seen in both in flaring state and quiescent state. At JD 2452699.5, NIR has shown anti-correlation with PCA data (this anti-correlation is responsible for lowering the correlation coefficients mentioned above). On March 01, 2003 observations could not be taken in NIR J band due to bad weather condition. So, NIR data for JD 2452700.5 is not present in the panel (c) of the figure 2.

\subsection{Spectral Energy Distribution (SED)}

The SED of Mrk 421 is plotted in the figure 5 in the form $\log \nu \mathrm{F}_{\nu}$ vs. $\log \nu$. All frequencies used here are observed frequencies. The synchrotron component of the SED was fitted using NIR and X-ray data with a parabolic function

$$
y=A x^{2}+B x+C
$$

the synchrotron peak frequency is determine by $\nu_{\text {peak }}=-\mathrm{B} / 2 \mathrm{~A}$. The $\nu_{\text {peak }}=$ 16.30 is calculated from the figure 5. It has been noticed for HBLs by Nieppola et al. (2006) that simple parabolic function produces some error. For HBLs, the synchrotron peak is expected close to soft X-ray band which decline to be very rapid. Thus in the real sense, the peak frequency will be slightly higher to the reported value.

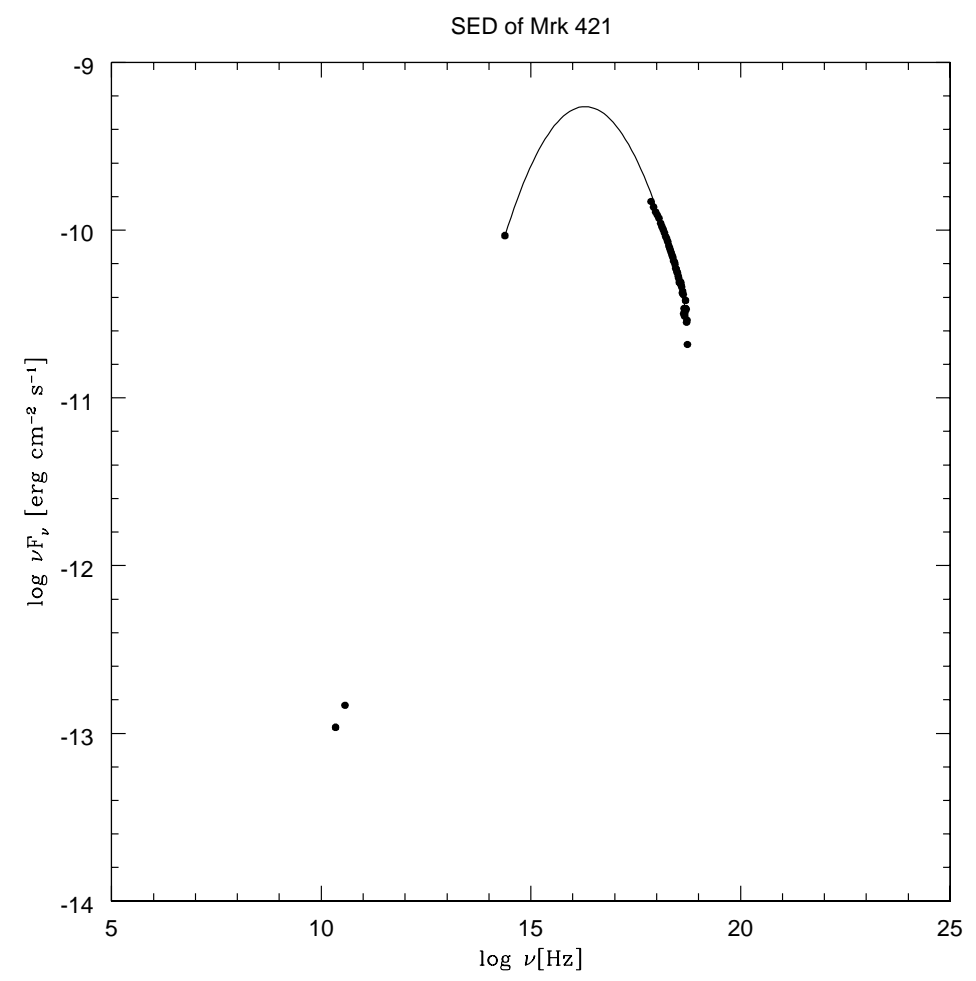

Fig. 5 Spectral energy distribution of the Mrk 421. 


\section{DISCUSSIONS}

We have found here the evidence of a possible correlation between X-ray and NIR wavelengths in Mrk 421 which is an HBL blazar.

So far, correlated X-ray and NIR variation was observed in a LBL blazar 3C 273, a flat spectrum radio quasar (FSRQ) (McHardy et al. 1999). For another LBL blazar AO $0235+164$, simultaneous radio and optical variability was reported during the optical outbursts occurred in 1975, 1979 and 1997 (MacLeod et al. 1976; Ledden et al. 1976; Rieke et al. 1976; Balonek \& Dent 1980; Webb et al. 2000) on the other hand no correlation was found between radio and optical emission of another LBL blazar S5 0716+714 (Ostorero et al. 2006). In a multiwavelength campaign of LBL blazar 3C 279 reported by (Wehrle et al. 1998), correlated variability in X-ray (PCA) and EGRET CGRO is seen without any time lag; UV leading gamma-ray by $\sim 2.5$ days. Since UV and gamma-ray data was less, so its reliability is doubtful.

Edelson et al. (1995) have reported correlated emission between X-ray, UV and optical emissions from PKS 2155-304 (an HBL BL Lac object). In another multi-wavelength campaign of PKS 2155-304 from 10 days data, Urry et al. 1997 have reported X-ray flare leading EUV flare by one day and UV flare by two days. McComb et al. (1995) and Maraschi et al. (1999) have reported simultaneous X-ray and $\gamma$-ray flares in Mrk 421 in two different campaigns. In other campaign of Mrk 421 in 1998 (Takahashi et al. 2000), complex variability, positive and negative lags were found which authors report may not be real, If the lag from both signs are real, these imply that particle acceleration and X-ray cooling timescales are similar. Katarzynski et al. (2003) show a well defined correlation between observed radio outburst in Mrk 421 with a corresponding X-ray outburst and a $\gamma$-ray flare in $\mathrm{TeV}$ range. In simultaneous $\mathrm{TeV}$ and optical observations of blazar 1406-076, optical flare leading gamma-ray flare by $\sim 1$ days (Wagner et al. 1995).

In a recent paper, Villata et al. (2006) have shown, in the historical radio and optical light curves variability behavior is different, while prominent and longlasting radio outbursts were visible at various radio frequencies, with higherfrequency variations preceding the lower-frequency ones. After that date, the optical activity increased and the radio flux is less variable, suggest that optical and radio emissions come from two separate and misaligned jet regions. The correlated emissions reported in several papers here, support models involving single populations of relativistic electrons responsible for the emission.

One of the important question is "Where does the NIR radiation originate"? There are three possibilities: (i) emission from circumstellar dust (ii) emission from the accretion disk (iii) synchrotron emission by relativistic electrons in the jet, the first two are external to the jet. The variability behavior is indicating the similar origin for NIR and X-ray emission. X-ray emission is originating from synchrotron radiation by electrons in this AGN as mentioned before.

From different multi-wavelength campaigns it has been noticed that variations on longer wavelengths are generally not seen during X-ray and $\gamma-$ ray flaring events (Tosti et al. 1998 and references therein). Ghisellini \& Maraschi (1996) have shown that the overall emission of the BL Lac Mrk 421 could be explained using a homogeneous SSC model, in which the equilibrium particle distribution is found balancing continuum injection, cooling and particle escape. In this, single population of relativistic electrons emit synchrotron radiation up to the UV or X-ray band and soft photons upto the IR-optical bands undergo upscattering with the most energetic electrons to form $\mathrm{TeV} \gamma$-rays. However, Blazejowski et al. (2005) have found $\mathrm{TeV}$ flares reached its peak days before 
X-ray flare during a giant flare or outburst in 2004. Spectral energy distribution (SED) generated by Blazejowski et al. (2005) was not fitted with one-zone synchrotron self-Compton (SSC) model but could be fitted well with additional zones.

Following Ghisellini \& Maraschi (1996), Marscher (1996) and other authors, we note that the lags in the multi-frequency light curves of Mrk 421 (of the order of hours between soft and medium X-ray photons, near simultaneous X-ray and $\gamma$-ray flares, IR leading X-rays by couple of days) require energy stratification in the source. Similar delays were noticed in $3 \mathrm{C} 279$ (IR leads X-ray by $0.75 \pm 0.25$ day) and in PKS 2155-304 (a day between EUV and X-rays, 2 days between UV and X-rays) and of the order of few hours between these (Edelson et al. 1995) as well. Frequency stratification and different time scales for the duration of these flares (shorter times for higher frequencies) are possible with the shock-in-jet model (Marscher 1996 and references therein).

Within the context of the shock-in-jet model of AGNs, we attribute the NIR emission component to the internal shock driven into the jet by the variation of the central engine. The correlated X-ray and NIR variability with time lag of few days is a strong probe of the jet emission not affected much by the environment of the surrounding medium. The anti-correlation seen in the NIR and soft X-ray light curve at JD 2452699.5 may be due to the reverse shock arising by the jet's collision with the surrounding medium.

Acknowledgements We thankfully acknowledge the referee for useful comments. The work of A. C. Gupta and J. H. Fan is supported by the National Natural Scientific Foundation of China (grant no. 10573005 and 10125313). We gratefully acknowledge the use of RXTE data from the public archive of GSFC/NASA. Also, we are grateful to all the members of PACT collaboration for their respective contributions.

\section{References}

Alexander, T., 1997, Astronomical Time Series, ed. D. Maoz, A. Sternberg \& E. M. Leibowitz, Dordrecht: Kluwer, p. 163

Balonek, T. J., Dent, W. A., 1980, ApJ, 240, L3

Bhat, P. N. et al., 2000, Bulletin of Astronomical Society of India, 20, 455

Blazejowski, M., Blaylock, G., Bond, I. H., et al. 2005, ApJ, 630, 130

Boone, L. M., Hinton, J. A., Bramel, D., et al. 2002, ApJ, 579, L5

Bose, D. et al., 2005, 29th International Cosmic Ray Conference Proceedings, 4, eds. B. S. Acharya et al., p. 343

Collmar, W., Bennett, K., Bloemen, H., et al. 1999, ApL\&C, 39, 57

Coppi, P. S. 1999, in workshop summary for the Cracow Workshop on Relativistic Jets in AGNs, ed. M. Ostrowski, M. Sikora, G. Madejski \& M. Begelman, Jagellonian University Press, p. 333

Edelson, R., Krolik, J., Madejski, G., et al., 1995, ApJ, 438, 120

Fan, J. H., \& Lin, R. G., 1999, ApJS, 121, 131

Ghisellini, G. \& Maraschi, L., 1996, ASP Conf. Series 110 on Blazar Continuum Variability, eds. H. R. Miller, J. R. Webb \& J. C. Noble, p. 436

Gothe, K. S. et al., 2000, Indian Journal of Pure \& Applied Physics, 38, 269

Gupta, A. C., Banerjee, D. P. K., Ashok, N. M., \& Joshi, U. C., 2004, A\&A, 422, 505

Heck D. et al., 1998, FZKA 6019 Forschungszentrum Karlsruhe 
Katarzynski, K., Sol, H., \& Kus, A. 2003, A\&A, 410, 101

Krawczynski, H. 2004, New Astronomy Rev., 48, 376

Ledden, J. E., Aller, H. D., Dent, W. A., 1976, Nature, 260, 752

Macomb, D. J., Akerlof, C. W., Aller, H. D., et al. 1995, ApJL, 449, L99

MacLeod, J. M., Andrew, B. H., Harvey, G. A., 1976, Nature, 260, 751

Makino, F., Tanaka, Y., Matsuoka, M., et al. 1987, ApJ, 313, 662

Majumdar, P., Acharya, B. S., Chitnis, V. R., et al. 2003, Astropaticle Physics, 18, 339

Maraschi, L., Fossati, G., Tavecchio, F., et al. 1999, ApJ, 526, L81

Marscher, A. P., 1996, ASP Conf. Series 110 on Blazar Continuum Variability, eds. H. R. Miller, J. R. Webb \& J. C. Noble, p. 248

McHardy, I., Lawson, A., Newsam, A., et al. 1999, MNRAS, 310, 571

Nieppola, E., Tornikoski, M., \& Valtaoja, E., 2006, A\&A, 445, 441

Ostorero, L., Wagner, S. J., Gracia, J., et al. 2006, A\&A, 451, 797

Petry, D., Bradbury, S. M., Konopelko, A., et al. 1996, A\&A, 311, L13

Punch, M., Akerlof, C. W., Cawley, M. F., et al. 1992, Nature, 358, 477

Rieke, G. H., Grasdalen, G. L., Kinman, T. D., et al. 1976, Nature, 260, 754

Sikora, M., \& Madejski, G., 2001, in AIP Conf. Proc. 558, High Energy Gamma-Ray Astronomy, ed. F. A. Aharonian \& H. J. Völk (new York: AIP), p. 463

Stein, W. A., O’Dell, S. L., \& Strittmatter, P. A., 1976, ARA\&A, 14, 173

Takahashi, T., Kataoka, J., Madejski, G. et al. 2000, ApJ, 542, L105

Taräsranta, H., Achren, J., Hanski, M., et al. 2004, A\&A, 427, 769

Taräsranta, H., Wiren, S., Koivisto, P., et al. 2005, A\&A, 440, 409

Thompson, D. J., Bertsch, D. L., Dingus, B. L., et al. 1995, ApJS, 101, 259

Tosti, G., Fiorucci, M., Luciani, M., et al. 1998, A\&A, 339, 41

Ulrich, M. H., Kinman, T. D., Lynds, C. R., et al. 1975, ApJ, 198, 261 eds. H. R. Miller, J. R. Webb \& J. C. Noble, p. 391

Urry, C. M., \& Padovani, P. 1995, PASP, 107, 803

Urry, C. M., Treves, A., Maraschi, L., et al. 1997, ApJ, 486, 799

Villata, M., Raiteri, C. M., Balonek, T. J., et al. 2006, A\&A, 453, 817

Wagner, S. J., Mattox, J. R., Hopp, U., et al. 1995, ApJ, 454, L97

Webb, J. R., Howard, E., Bentez, E., et al. 2000, AJ, 120, 41

Wehrle, A. E., Pian, E., Urry, C. M., et al. 1998, ApJ, 497, 178

Xie, G.-Z., Lu, R.-W., Zhou, Y., et al. 1988, A\&AS, 72, 163

This manuscript was prepared with the ChJAA LATEX macro v1.2. 